\title{
RAMÓN GONZÁLEZ RUIZ, DÁMASO IZQUIERDO ALEGRÍA Y ÓSCAR LOUREDA LAMAS (EDS.) LA EVIDENCIALIDAD EN ESPAÑOL: TEORÍA Y DESCRIPCIÓN. IBEROAMERICANA VERVUERT, 2016. LINGÜÍSTICA IBEROAMERICANA VOL. 60. 350 PAGINAS
}

\author{
Karolina GrZech \\ SOAS, Universidad de Londres \\ kg7@soas.ac.uk
}

En los últimos años, la categoría de la evidencialidad se ha convertido en un tema muy popular entre los lingüistas. La investigación de los sistemas evidenciales ha ido cogiendo velocidad desde la publicación del influyente trabajo de Aikhenvald: una encuesta interlingüística sobre las expresiones evidenciales (2004). Sin embargo, esta obra ha establecido una división teórica de mayor importancia, definiendo la evidencialidad como una categoría gramatical. Este planteamiento ha excluido del campo de estudios evidenciales a todas aquellas lenguas que tan solo poseen 'estrategias evidenciales' (Aikhenvald 2004, cap. 4), o sea, que no gocen de un sistema gramaticalmente obligatorio cuya principal función sea expresar valores evidenciales. Según este planteamiento, tanto el español como otras lenguas europeas se consideran 'no-evidenciales'.

La perspectiva impulsada por Aikhenvald ha dejado su impronta en los estudios teóricos y descriptivos de la evidencialidad, introduciendo una separación entre aquellas lenguas que gramaticalizan la categoría y las que no lo hacen. No obstante, esta dicotomía ha sido cuestionada por los análisis más recientes, centrados en el rol comunicativo de la evidencialidad (vid., p.ej., Nuckolls \& Michael, 2014). Dichos estudios han demostrado que estudiar tan solo las expresiones gramaticalizadas y obligatorias de la evidencialidad limita nuestro entendimiento de su función comunicativa. Por lo tanto, para poder comprender y analizar su papel en la comunicación, es más adecuado definir la evidencialidad como una categoría semántico-funcional. Este planteamiento permite suprimir la división entre 'evidencialidad propia' y las 'estrategias evidenciales', ya que ambos tipos de expresiones cumplen similares funciones discursivas.

Los editores del libro La evidencialidad en español: teoría y descripción parten de esta premisa, integrando en el volumen los análisis de diferentes tipos de expresiones catalogadas como evidenciales en los estudios sobre esta categoría en español. Ramón González Ruiz y Dámaso

Para citar esta reseña / To cite this book review: Grzech, Karolina (2017). Reseña de Ramón González Ruiz, Dámaso Izquierdo Alegría y Óscar Loureda Lamas (eds.) (2016): La evidencialidad en español: teoría y descripción. Iberoamericana Vervuert. Lingüística Iberoamericana vol. 60. (350 paginas). ELUA, 31: 393-396. doi:10.14198/ELUA2017.31.24

Enlace / Link: http://dx.doi.org/10.14198/ELUA2017.31.24 
Izquierdo Alegría de la Universidad de Navarra, y Óscar Loureda Lamas de la Universidad de Heidelberg, son expertos en el estudio del español desde una perspectiva evidencialista. Su brillante capítulo de introducción resume las diferentes corrientes de los estudios evidenciales desde la década de los 80 , cuando surgió un interés más extendido por la categoría.

El capítulo proporciona una aproximación histórica a los estudios evidenciales, define los conceptos básicos y aproxima a los lectores a la historia del concepto de evidencialidad en el ámbito de la lingüística hispánica. Si bien los autores parten de una definición de la evidencialidad como una categoría funcional y no gramatical, no imponen a los contribuidores una definición uniforme de esta noción. En consecuencia, a lo largo de los diversos capítulos, la evidencialidad se define tanto en sentido 'ancho', que incluye la modalidad epistémica, como en sentido 'estrecho', que la excluye. Esa diversidad de perspectivas permite, de acuerdo con la intención de los editores, que el libro sea 'un espejo representativo del actual panorama de estudios teóricos y descriptivos evidencialistas en la lingüística hispánica' (González Ruiz et al. 2016: 34).

La monografía está dividida en cinco partes. La primera concierne a las cuestiones teóricas relacionadas con la noción de evidencialidad y su aplicación en español. La segunda parte trata de expresiones de la evidencialidad mediante adverbios y partículas discursivas. En la tercera, se analizan los significados evidenciales de los verbos y adverbios modales, y en la cuarta, la expresión de la evidencialidad a través de los tiempos verbales. En el último apartado se presenta un enfoque contrastivo al estudio de la evidencialidad. En lo que sigue, resumiré brevemente las contribuciones contenidas en cada una de las partes.

La parte teórica comienza con un capítulo de Mercedes González Vásquez, de la Universidad de Vigo. Resume las diferentes maneras de definir la evidencialidad, tomando en cuenta también el nivel comunicativo en el cual los evidenciales modifican el acto de habla. Por consiguiente, ofrece un resumen de las formas en las que se expresa la evidencialidad en español, abordando medios léxicos y estrategias gramaticales. En el segundo artículo de la parte teórica, Marta Albelda Marco (Universitat de València) explora la relación entre la evidencialidad y la atenuación. A modo de introducción, analiza las relaciones entre la atenuación y la evidencialidad, por un lado, y la modalidad epistémica, por otro. Su aportación consiste en un estudio de campo en el que examina cinco expresiones evidenciales y sus funciones atenuantes, ilustrando ampliamente sus observaciones con los ejemplos del corpus de datos escritos y orales.

La segunda parte, dedicada a los adverbios y partículas discursivas, empieza con la contribución de Antonio Briz, de la Universitat de València y Grupo Val.Es.Co. Briz explora la relación entre la evidencialidad, la atenuación y la intensificación, centrándose en el uso de las partículas discursivas. A continuación, Elisa González Ramos (Universidad de Zaragoza) trata la restricción del compromiso con la verdad en el caso de las locuciones adverbiales por lo visto y al parecer. La autora describe el valor evidencial y modal de dichas oraciones, explicando cómo su uso remite a la interpretación evidencial indirecta, tanto inferencial como reportativa.

En el siguiente capítulo, Mercedes Marcos Sánchez (Universidad de Salamanca) explora la relación entre subjetividad e intersubjetividad y el uso de los marcadores evidenciales formados a partir del verbo ver. Define la evidencialidad como la 'justificación epistémica', de acuerdo con la propuesta de Boye (2012). Usando esta noción, el capítulo propone un análisis multidimensional de los marcadores parentéticos con ver, aproxi- 
mando a los lectores las nociones de subjetividad e intersubjetividad, que gozan de una creciente importancia en el estudio contemporáneo de los sistemas evidenciales y epistémicos (vid., p.ej., Verhagen, 2005; Traugott, 2010; Gipper, 2011).

Seguidamente, Teresa $\mathrm{M}^{\mathrm{a}}$ Rodríguez Ramalle, de la Universidad Complutense de Madrid, analiza el rol de las partículas evidenciales en el desarrollo del discurso reformulativo. Se enfoca en las locuciones basadas en el que: que, sea que y así que, y muestra cómo pueden llegar a introducir enunciados basados en la inferencia del hablante. En el último capítulo de la segunda parte, Pedro Gras, de la Universidad de Antwerpen, propone un análisis de los valores citativos del que inicial átono, ilustrando sus análisis con los ejemplos del corpus del español coloquial hablado del grupo Val.Es.Co (Briz \& Grupo Val.Es.Co, 2002).

La tercera parte se centra en los verbos y adverbios modales. La contribución de Bert Cornillie, de la Universidad Católica de Leuven, explora los significados evidenciales de seis verbos auxiliares: deber, tener que, parecer, resultar, amenazar y prometer. Se basa en una serie de criterios formales, distribucionales y semánticos para respaldar su análisis como marcadores evidenciales de la inferencia, y se interroga acerca de su valor (inter) subjetivo. En el siguiente capítulo, Sergi Torner (Universitat Pompeu Fabra) aborda los adverbios evidenciales aparentemente, evidentemente, obviamente, supuestamente y visiblemente. Analiza tanto el valor evidencial de dichos adverbios como su rol atenuador o reforzador con respecto al enunciado.

La cuarta parte analiza lo que más se aproxima a la 'evidencialidad propia' según la definición de Aikhenvald (2004): las expresiones de la evidencialidad a través de los tiempos verbales. María Marta García Negroni de la Universidad San Andrés/Universidad de Buenos Aires/COINCET estudia los usos citativos del futuro morfológico y del futuro perifrástico. Usa para su análisis el marco de la teoría polifónico-argumentativa (Ducrot, 1984), y se basa en datos provenientes tanto del español peninsular como del argentino. En el siguiente capítulo, Susana Azpiazu (Universidad de Salamanca) analiza el significado evidencial del pretérito perfecto compuesto en español, a partir del discurso coloquial en el español peninsular en comparación con el español andino. Demuestra que, en el caso del español peninsular, el significado evidencial es una implicatura asociada con el uso del pretérito perfecto compuesto. Sin embargo, también prueba que esta interpretación no puede sustentarse para el español andino y otras variantes habladas en América Latina.

La última parte del libro aporta una aproximación hacia un enfoque contrastivo en los estudios de la evidencialidad en español. La sección está compuesta por un solo artículo: la contribución de Juana I. Marín Arrense, de la Universidad Complutense de Madrid, quien compara la evidencialidad en el discurso periodístico entre el castellano y el inglés. Empieza con una amplia introducción teórica, aproximando a los lectores los diferentes tipos de evidencialidad inferencial indirecta que la autora considera en el análisis. Después realiza un análisis comparativo de textos periodísticos de dos subgéneros: editoriales y columnas de opinión. El capítulo termina con un resumen de las similitudes y diferencias en el uso de las expresiones modales epistémicas y evidenciales (inferenciales y reportativas), tanto entre ambos subgéneros como entre el español y el inglés.

En su conjunto, la monografía constituye una aportación única, novedosa y muy actual en los estudios de la evidencialidad en español. Cabe destacar que los diferentes autores, además de describir las diversas expresiones y estrategias evidenciales en español, abarcan 
también los debates pertinentes de las últimas décadas: relación de la evidencialidad con modalidad epistémica (Briz; González Ramos; Marín Arrense), con la atenuación (Albelda; Briz; Torner), la (inter)subjetividad (Marcos Sánchez; Cornillie; Marín Arrense) o la epistemicidad (Marín Arrense).

El libro sin duda será de utilidad para aquellos estudiantes que quieran iniciarse en el estudio de la evidencialidad. También puede servir como un recordatorio útil para los que tengan un conocimiento más amplio del tema o busquen un resumen del estado de la cuestión dentro de la lingüística hispánica. Además, es una referencia muy valiosa para todos aquellos que se interesan por las lenguas con evidencialidad gramaticalizada y buscan nuevas inspiraciones teóricas y metodológicas.

\section{Referencias bibliográficas}

Aikhenvald, Alexandra Y. 2004. Evidentiality. Oxford: Oxford University Press.

Boye, Kasper. 2012. Epistemic Meaning, A Crosslinguistic and Functional-Cognitive Study. Berlin, Boston: De Gruyter Mouton.

Briz, Antonio \& Grupo Val.Es.Co. 2002. Corpus de conversaciones coloquiales. Anejo de la revista Oralia. Arcos/Libros.

Ducrot, Oswald. 1984. Le Dire et le dit. Paris: Editions de Minuit.

Gipper, Sonja. 2011. Evidentiality and Intersubjectivity in Yurakaré: an Interactional Account. Nijmegen: MPI

González Ruiz, Ramón, Oscar Loureda Lamas \& Dámaso Izquierdo Alegría. 2016. Un acercamiento a los fundamentos de la evidencialidad y a su recepción y tratamiento en la lingüística hispánica. En González Ruiz, Ramón, Oscar Loureda Lamas \& Dámaso Izquierdo Alegría (eds.), La evidencialidad en español: teoría y descripción, 9-45. (Lingüística Iberoamericana 60). Madrid: Iberoamericana; Frankfurt am Main: Vervuert.

Nuckolls, Janis \& Lev Michael. 2014. Evidentiality in interaction. Amsterdam, Philadephia: John Benjamins.

Traugott, Elizabeth Closs. 2010. (Inter)subjectivity and (inter)subjectification: A reassessment. En Davidse, Kristin, Lieven Vandelanotte \& Hubert Cuyckens (eds.), Subjectification, Intersubjectification and Grammaticalization, vol. 66, 29-74. Berlin, New York: De Gruyter Mouton.

Verhagen, Arie. 2007. Constructions of Intersubjectivity: Discourse, Syntax, and Cognition. Oxford: Oxford University Press. 